\section{A method for the precise switching of speech}

\author{
JOHN A. RUPF* and GEORGE W. HUGHES \\ Purdue University, Lafayette, Indiana 47907 \\ and \\ STEPHEN V. ROWSON and KENNETH F. RUDER \\ University of Kansas, Lawrence, Kansas 66044
}

A simple procedure by which a speech signal may be switched on or off (or from one ear to the other) at a precise instant of time is described. The speech sample is first recorded on a magnetic tape loop. A reference pulse is then recorded preceding the speech signal. This reference pulse triggers an adjustable time delay which in turn operates an elentronic switch at an appropriate time.

In performing listening experiments involving various temporal aspects of speech, it is often necessary to turn speech on or off (or to switch speech from one ear to the other) at a precise instant of time within the duration of the speech waveform. The procedure below describes a straightforward method for obtaining a dual-channel tape such that the portion of speech up to a desired transition point is recorded on one channel and the remainder on a second channel. The accuracy with which the transition or switching point can be positioned relative to a selected position in the speech waveform depends upon the specific equipment used. With the equipment used by the authors, ${ }^{1}$ an accuracy of $\pm 0.5 \mathrm{msec}$ was obtained.

The procedure consists essentially of recording the speech segment to be studied on one channel of a dual-channel tape loop, and recording a reference pulse on the other channel of the loop at a point in time earlier than the desired switching or transition point in the speech segment. The reference pulse, via an appropriate time delay, triggers an electronic switch which then switches the speech.

The details of the procedure follow: A tape loop is mounted on the two-track tape recorder, No. 1 (see Fig. 1), and the speech sequence to be studied is recorded on Channel $A$. syllable) then an alternate method for recording the reference pulse should should be capable of providing delays of up to at least 1 sec long.

If the desired transition point is very near the beginning of the speech

Then the recorder is set to play back Channel B. A reference pulse is recorded on Channel B by manually fraction of a second before the desired reint. The position checked by listening to Channels $A$ should be heard before the desir maximum delay obtainable from the variable time-delay generator referred

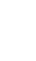
tre be used. In this case, the loop is turned over after recording the speech so that the speech plays backward on Channel B. The reference pulse is then recorded on Channel $A$ a fraction of a second after the desired transition point. Changing the loop back to its original position allows the pulse position to be checked as described above. The pulse amplitude should not exceed the maximum voltage rating of the tape-recorder input, but should be large enough to produce an audible click. A pulse width of 0.2 msec yields satisfactory results.

Once a suitable reference pulse has been recorded, the equipment is set up as shown in Fig. 2. The output of Channel A (the speech signal) from Recorder No. 1 is connected to the A input of Channel 1 and the $B$ input of Channel 2 of an electronic switch. The Channel 1 and 2 outputs from the switch are in turn connected to the $A$ and B inputs, respectively, of Recorder No. 2. The Channel A output of Recorder No. 1 is monitored on one channel of a storage oscilloscope.

Channel B output from Recorder No. 1 (the reference pulse) is connected to a variable time-delay generator. The reference pulse triggers the time-delay generator which in turn (after an appropriate delay) triggers the pulse generator which operates the electronic switch. Since the reference pulse occurs at a fixed point in time with respect to the speech signal, the time at which the switching occurs can be adjusted by varying the amount of time delay between the occurrence of 
the reference pulse and the triggering of the electronic switch. When the electronic switch and Recorder No. 2 are connected as shown in Fig. 2, the speech is switched from Channel $A$ to Channel $B$ (or vice versa, depending on the initial state of the electronic switch) of the tape recorder.

Operation of the electronic switch at the desired transition point is accomplished by continuously cycling the tape loop on Recorder No. 1 and adjusting the time-delay generator while monitoring the position of the switch operation relative to the desired transition point in the speech signal. Monitoring of the transition point may be either aural or visual, or both. To monitor aurally, stereo headphones are connected to the Channel 1 and 2 outputs of the electronic switch. The delay of the time-delay generator is then varied until the speech switches between ears at the desired transition point in the speech sequence. Increasing the delay of the time-delay generator causes the transition pulse to occur at a later time. The electronic switch must be reset each cycle after it has been triggered by the pulse generator.

Visual monitoring provides an alternate method of placing the position of the transition point. When connected as shown in Fig. 2, one trace on the oscilloscope screen displays the speech waveform while the other trace displays the pulse which operates the electronic switch. The oscilloscope is triggered externally by the reference pulse from Channel $B$ of Recorder No. 1. The time-delay generator is adjusted until the pulse displayed on the oscilloscope occurs at the transition point. For maximum accuracy, an oscilloscope with delayed sweep capability should be used so that a fairly small increment of time containing the desired transition point can be made to cover the entire width of the CRT face.

The basic accuracy of the system is limited by the repeatability accuracy of the variable time-delay generator and tape-recorder speed stability. Any professional-quality dual-channel tape recorder should provide satisfactory performance, as should any high-quality electronic switch. The pulse generator and time-delay generator should be selected such that the pulse generator is capable of recording on the tape a reference pulse of sufficient magnitude to trigger the time-delay generator, which in turn should provide a pulse capable of triggering the electronic switch.

\section{NOTE}

1. The method described herein does not depend on specific models of equipment: however, the apparatus used by the authors included an Ampex 354 tape recorder, a Tektronix 564 oscilloscope with Type $3 \mathrm{~B} 3$ time base, a Grason-Stadler $829 \mathrm{E}$ electronic switch, and two General Radio Type 1217-C unit pulse generators used as a time-delay generator.

* Present address: Department of Electrical Engineering. University of Kansas, Lawrence, Kansas 66044. 